\title{
Comment on: "Association of Early Systemic Corticosteroid Therapy with Mortality in Patients with Stevens-Johnson Syndrome or Toxic Epidermal Necrolysis: A Retrospective Cohort Study Using a Nationwide Claims Database"
}

\author{
André Cerejeira ${ }^{1}$ (D)
}

Published online: 12 September 2019

(c) Springer Nature Switzerland AG 2019

To the Editor:

I read with great interest the article "Association of Early Systemic Corticosteroid Therapy with Mortality in Patients with Stevens-Johnson Syndrome or Toxic Epidermal Necrolysis: A Retrospective Cohort Study Using a Nationwide Claims Database" [1].

In this paper, propensity score matching (PSM) was used to reduce imbalance in the empirical distribution of the pretreatment confounders between the treated and control groups.

Matching can be thought of as a technique for finding approximately ideal experimental data hidden within an observational data set. PSM is the most popular matching method worldwide, perhaps even "the most developed and popular strategy for causal analysis in observational studies" [2].

However, according to a recently published article (appropriately entitled "Why Propensity Scores Should Not Be Used for Matching"), PSM often accomplishes the opposite of its intended goal-a problem referred to as "the PSM paradox" [3]. In fact, PSM approximates an experimental design with lower standards than necessary, thus failing to use all of the information available and generating higher

This comment refers to the article available at https://doi. org/10.1007/s40257-019-00443-9.

Reply to this letter to the editor is available at https://doi. org/10.1007/s40257-019-00473-3.

André Cerejeira

andrecerejeira_@hotmail.com

1 Department of Dermatology and Venereology, Centro Hospitalar Universitário São João EPE, Alameda Prof. Hernâni Monteiro, 4200-319 Porto, Portugal levels of imbalance, inefficiency, model dependence and statistical bias.

The core insight behind PSM is to analyze an observational data set by approximating as closely as possible to a completely randomized experiment. However, as the authors demonstrated in this paper, approximating to a fully blocked randomized experiment can be substantially better.

Other matching methods will usually achieve lower levels of imbalance than PSM, even given the same number of observations pruned, and do not generate a similar paradox until much later in the pruning process. Therefore, matching should remain a highly recommended method of causal inference, but other matching methods might be preferable.

\section{Compliance with Ethical Standards}

Funding No funding was received for the preparation of this letter.

Conflict of interest André Cerejeira have no conflicts of interest that are directly relevant to the content of this letter.

\section{References}

1. Morita K, et al. Association of early systemic corticosteroid therapy with mortality in patients with Stevens-Johnson syndrome or toxic epidermal necrolysis: a retrospective cohort study using a nationwide claims database. Am J Clin Dermatol. 2019;20(4):579-92.

2. Pearl J. The foundations of causal inference. Sociol Methodol. 2010;40(1):75-149.

3. King G, Nielsen R. Why propensity scores should not be used for matching. Polit Anal 2016; 1-20. 